\title{
Totally geodesic surfaces with arbitrarily many compressions
}

\author{
PRADTHANA JAIPONG
}

\begin{abstract}
A closed totally geodesic surface in the figure eight knot complement remains incompressible in all but finitely many Dehn fillings. In this paper, we show that there is no universal upper bound on the number of such fillings, independent of the surface. This answers a question of Ying-Qing Wu.
\end{abstract}

$57 \mathrm{~N} 10,57 \mathrm{~N} 25 ; 57 \mathrm{~N} 50$

\section{Introduction}

Let $M$ be a compact, connected, irreducible, orientable 3-manifold with torus boundary $\partial M$. A slope on $\partial M$ is an isotopy class of simple closed curves on $\partial M$. We use $\Delta(\alpha, \beta)$ to denote the absolute value of the algebraic intersection number between the slopes $\alpha$ and $\beta$. Culler, Gordon, Luecke and Shalen [4] showed that if $F$ is a closed, orientable, embedded, incompressible surface in $M$ with no incompressible annulus joining $F$ and $\partial M$, and $F$ compresses in the Dehn fillings $M(\alpha)$ and $M(\beta)$, then $\Delta(\alpha, \beta) \leq 2$. In [17], Wu improved this to $\Delta(\alpha, \beta) \leq 1$, and hence $F$ remains incompressible in $M(\gamma)$ for all but at most three slopes $\gamma$.

If one drops the assumption that $F$ be embedded, the previous theorem is not true; see Leininger [7]. However, for hyperbolic $M$ such a surface $F$ can compress in at most finitely many Dehn fillings $M(\gamma)$; see Bart [1]. In fact in [19], Wu showed that there is a bound on the number of fillings in which $F$ can compress depending only on the genus of $F$, and not on the manifold $M$. Wu has asked whether there is any universal bound, independent of $F$, for this number [18, Question 6.6]. In this paper, we prove that no such universal bound exists. More precisely we prove:

Theorem 1.1 There exists a compact, connected, orientable, 3-manifold $M$ with torus boundary and hyperbolic interior having the following properties. Given any positive integer $n$, there exist $n$ distinct slopes $\alpha_{1}, \ldots, \alpha_{n}$ and infinitely many pairwise noncommensurable closed, orientable, immersed, incompressible surfaces $F \leftrightarrow M$, with no incompressible annulus joining $F$ and $\partial M$, such that $F$ compresses in $M\left(\alpha_{i}\right)$ for all $i=1, \ldots, n$. 
The manifold in Theorem 1.1 is $M_{8}$, the exterior of the figure eight knot in $S^{3}$. Our proof involves a careful analysis of a construction of closed, immersed, totally geodesic surfaces in $M_{8}$ which compress in $M_{8}(\gamma)$ for some specific $\gamma$. In particular, we inspect the proof of the following theorem of Leininger [7].

Theorem 1.2 Suppose $4 \mid p$ and $3 \nmid p$. Then for any $q$ that is relatively prime to $p$ there exists infinitely many noncommensurable, closed, immersed, totally geodesic surfaces in $M_{8}$ which compress in $M_{8}(p / q)$.

This paper is organized as follows: Section 2 contains a few definitions and constructions from 3-dimensional topology necessary for our work. We then give a brief review of some basic definitions and facts concerning hyperbolic 3-manifolds in Section 3. Section 4 contains the various constructions of totally geodesic surfaces and theorems on compressing totally geodesic surfaces. Section 5 includes definitions and facts from number theory and quadratic forms, and we prove the main technical theorem needed for the proof of Theorem 1.1. In Section 6, we prove Theorem 1.1.

Acknowledgments The author is indebted to Professor Christopher Leininger for key discussions and for his careful reading of early versions of this manuscript. The author would like to thank Professor Kenneth Williams for his helpful suggestion on using quadratic forms. The author also appreciates the support from NSF during the Spring and Summer 2010.

\section{3-dimensional topology}

In this section we recall some definitions and facts from 3-dimensional topology. For more details, see Benedetti and Petronio [2], Rolfsen [14] and Thurston [16].

Let $M$ be a compact, orientable 3-manifold with a torus boundary $\partial M \cong T^{2}$ and let $\pi_{1}(\partial M) \cong \pi_{1}\left(T^{2}\right) \cong \mathbb{Z} \oplus \mathbb{Z}$ be generated by $\lambda$ and $\mu$. A slope on $\partial M$ is an isotopy class of simple closed curves on $\partial M$, and can be uniquely associated (up to inverses) with a primitive element $\alpha=\lambda^{p} \mu^{q} \in \pi_{1}(\partial M)$. Primitivity implies $p, q$ are relatively prime and so the set slopes are in a one-to-one correspondence with $\mathbb{Q} \bigcup\{\infty\}$, where $\lambda^{p} \mu^{q}$ corresponds to $p / q$ in the lowest terms. We write $\alpha=p / q$ (with $\infty=1 / 0$ ). If $\alpha=p / q$ and $\sigma=r / s$ are two slopes in $\pi_{1}(\partial M)$, then the distance between $\alpha$ and $\sigma$ is given by $\Delta(\alpha, \sigma)=|p s-q r|$.

Now let $\alpha$ be a slope on $\partial M, S^{1} \times D^{2}$ be a solid torus and $\mu_{0}=\{*\} \times \partial D^{2}$ be a meridional curve on $\partial\left(S^{1} \times D^{2}\right)$. We form a closed 3-manifold by $\alpha-D e h n$ filling on $\partial M$ by attaching $S^{1} \times D^{2}$ to $M$ identifying $\partial\left(S^{1} \times D^{2}\right)$ with $\partial M$ so that $\alpha$ is 
identified with $\mu_{0}$. The resulting space, denoted by $M(\alpha)$, is a closed 3-manifold depending only on $\alpha$ up to homeomorphism of $M(\alpha)$.

Let $F$ be a closed, connected, orientable surface which is not homeomorphic to a 2 -sphere. We say that an immersion $f: F \longrightarrow M$ is an incompressible surface if the induced map $f_{*}: \pi_{1}(F) \longrightarrow \pi_{1}(M)$ is injective, and compressible, otherwise. A surface $f: F \longrightarrow M$ is essential if it is incompressible and is not homotopic into $\partial M$. We say $f: F \longrightarrow M$ is acylindrical if no element of $f_{*}\left(\pi_{1}(F)\right)$ is peripheral, that is, conjugate into $\pi_{1}(\partial M)$. Equivalently, there is no annulus in $M$ joining a nontrivial loop in $F$ to a loop in $\partial M$.

The manifold $M$ we are interested in here will be the interior of compact manifold $\bar{M}$ with torus boundary. We will write $M(\alpha)$ for $\bar{M}(\alpha)$, and will refer to $\partial M$ for $\partial \bar{M}$. We will generally not distinguish between $M$ and $\bar{M}$ when no confusion arises.

The figure eight knot $K \subset S^{3}$ is the knot whose projection is shown in Figure 1. The manifold we will analyze is $M_{8}=S^{3}-K$ which is the interior of a compact manifold with torus boundary. In the next two sections, we will describe this manifold in more detail.

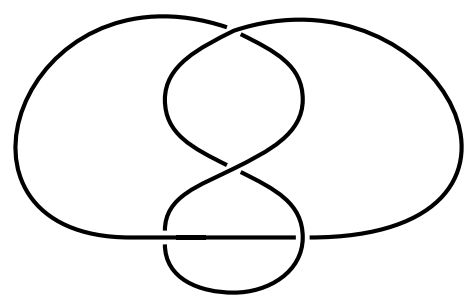

Figure 1: The figure eight knot

\section{Hyperbolic 3-manifolds}

Here we review some of the background concerning hyperbolic 3-manifolds. See Maclachlan [10] and Ratcliffe [11] for more details.

Let us consider the upper half space

$$
\mathbb{H}^{3}=\{(z, t) \in \mathbb{C} \times \mathbb{R} \mid t>0\}
$$

endowed with the complete Riemannian metric

$$
d s^{2}=\frac{|d z|^{2}+d t^{2}}{t^{2}}
$$


which is a model for hyperbolic 3 -space. The boundary at infinity $\partial \mathbb{H}^{3}$ is $\widehat{\mathbb{C}}=$ $(\mathbb{C} \times\{0\}) \cup\{\infty\}$. The group of all orientation-preserving isometries of $\mathbb{H}^{3}$ is isomorphic to $\operatorname{PSL}_{2}(\mathbb{C})$ acting by conformal extension of Möbius transformation on $\widehat{\mathbb{C}}$.

As a convention, since $\mathrm{PSL}_{2}(\mathbb{C}) \cong \mathrm{SL}_{2}(\mathbb{C}) / \pm I$, whenever we refer to a matrix for an element in $\mathrm{PSL}_{2}(\mathbb{C})$, we really mean one of the corresponding matrices in $\mathrm{SL}_{2}(\mathbb{C})$ under the quotient homomorphism. A subgroup $\Gamma$ of $\mathrm{PSL}_{2}(\mathbb{C})$ is said to be a Kleinian group if the induced topology on $\Gamma$ is the discrete topology. Equivalently, $\Gamma$ acts properly discontinuously on $\mathbb{H}^{3}$.

Throughout this paper, we will consider $\Gamma$ a torsion-free Kleinian group. Let $M_{\Gamma}=$ $\mathbb{H}^{3} / \Gamma$ be the quotient hyperbolic 3-manifold with its induced metric, so we have $\pi_{1}\left(M_{\Gamma}\right) \cong \Gamma$. We say that $\Gamma$ is cocompact or has finite covolume if $M_{\Gamma}$ is compact or has finite total volume, respectively.

As is shown in Riley [13] (see also Maclachlan [10] and Ratcliffe [11]), $M_{8} \cong \mathbb{H}^{3} / \Gamma_{8}$, and $\pi_{1}\left(M_{8}\right) \cong \Gamma_{8}$, where

$$
\Gamma_{8}=\left\langle\left(\begin{array}{ll}
1 & 1 \\
0 & 1
\end{array}\right),\left(\begin{array}{cc}
1 & 0 \\
-\omega & 1
\end{array}\right)\right\rangle,
$$

with $\omega=(-1+\sqrt{-3}) / 2$. We note $\mathbb{Z}[\omega]=\mathcal{O}_{3}$, the ring of integers in the quadratic number field $\mathbb{Q}(\sqrt{-3})$. Furthermore $\Gamma_{8}$ has index 12 in $\operatorname{PSL}_{2}\left(\mathcal{O}_{3}\right)$.

\section{Surfaces in hyperbolic 3-manifolds}

In this section we collect some of definitions and facts concerning surface in hyperbolic 3-manifolds. See Long and Reid [8], Maclachlan [10], and Thurston [16] for more details.

\subsection{Totally geodesic surfaces in $M_{\Gamma}=\mathbb{H}^{3} / \Gamma$}

All closed, orientable, immersed, totally geodesic surfaces in $M_{\Gamma}=\mathbb{H}^{3} / \Gamma$ arise as follows.

Let $\mathcal{C}$ be any circle in $\widehat{\mathbb{C}}$, that is, a circle or line in $\mathbb{C}$. For any subgroup $\Gamma \subseteq \mathrm{PSL}_{2}(\mathbb{C})$, define

$$
\operatorname{Stab}_{\Gamma}(\mathcal{C})=\{g \in \Gamma \mid g(\mathcal{C})=\mathcal{C} \text { and } g \text { preserves the components of } \widehat{\mathbb{C}} \backslash \mathcal{C}\} .
$$

For any circle $\mathcal{C}$, a discrete subgroup of $\operatorname{Stab}_{\mathrm{PSL}_{2}(\mathbb{C})}(\mathcal{C})$ is called a Fuchsian group. Because of the transitive action of $\mathrm{PSL}_{2}(\mathbb{C})$ on circles in $\widehat{\mathbb{C}}$, there exists $g \in \mathrm{PSL}_{2}(\mathbb{C})$ such that $g(\mathcal{C})=\widehat{\mathbb{R}}=\mathbb{R} \bigcup\{\infty\}$, and hence $g \operatorname{Stab}_{\mathrm{PSL}_{2}(\mathbb{C})}(\mathcal{C}) g^{-1}=\operatorname{PSL}_{2}(\mathbb{R})$. 
Any circle $\mathcal{C}$ in $\widehat{\mathbb{C}}$ bounds a hyperbolic plane $P_{\mathcal{C}} \cong \mathbb{H}^{2}$ embedded totally geodesically in $\mathbb{H}^{3}$. If $\Gamma^{\prime}=\operatorname{Stab}_{\Gamma}(\mathcal{C})$ is a torsion free Fuchsian group, we obtain a hyperbolic surface $S_{\Gamma^{\prime}}=P_{\mathcal{C}} / \Gamma^{\prime}$ with $\pi_{1}\left(S_{\Gamma^{\prime}}\right) \cong \Gamma^{\prime}$.

Let $\Gamma$ be a finite covolume torsion free Kleinian group such that there exists a circle $\mathcal{C} \subset \widehat{\mathbb{C}}$ for which $\Gamma^{\prime}=\operatorname{Stab}_{\Gamma}(\mathcal{C})$ has finite coarea. One can check that this induces a proper totally geodesic incompressible immersion

$$
S_{\Gamma^{\prime}} \cong P_{\mathcal{C}} / \Gamma^{\prime} \leftrightarrow M_{\Gamma} \cong \mathbb{H}^{3} / \Gamma
$$

Let us write $\mathcal{C}_{D}$ to denote a circle centered at the origin with radius $D \in \mathbb{Z}^{+}$. Consider the subgroup of $\Gamma_{8}$,

$$
\Gamma_{D}=\operatorname{Stab}_{\Gamma_{8}}\left(\mathcal{C}_{D}\right)=\left\{\gamma \in \Gamma_{8} \mid \gamma\left(\mathcal{C}_{D}\right)=\mathcal{C}_{D}\right\},
$$

which always has finite coarea [9].

We say $\Gamma_{D}, \Gamma_{D^{\prime}}$ are commensurable if there exists an element $g \in \Gamma_{8}$ such that $\left(g \Gamma_{D} g^{-1}\right) \cap \Gamma_{D^{\prime}}$ is a finite index subgroup in both $g \Gamma_{D} g^{-1}$ and $\Gamma_{D^{\prime}}$. The following is a consequence of arithmeticity (see Leininger [7] and Reid [12]).

Theorem 4.1 For each positive integer $D \equiv 2(\bmod 3), \Gamma_{D}$ is a cocompact Fuchsian group and therefore $S_{D}=P_{D} / \Gamma_{D} \rightarrow M_{8}$ is a closed totally geodesic surface, which is in particular acylindrical. Moreover, $\Gamma_{D}$ and $\Gamma_{D^{\prime}}$ are commensurable in $\Gamma_{8}$ if and only if $D=D^{\prime}$.

\subsection{Compressing totally geodesic surfaces}

For the proof of the main theorem, we analyze the construction used in the proof of Theorem 1.2. The details of its proof are in [7].

The construction is started by considering $\pi_{1}\left(\partial \bar{M}_{8}\right) \cong \mathbb{Z} \oplus \mathbb{Z}$ which is generated by the standard meridian-longitude $\mu$ and $\lambda$.

For any given integers $p$ and $q$ with $\operatorname{gcd}(p, q)=1$, set $\sigma=\lambda^{p} \mu^{q} \in \pi_{1}\left(\partial \overline{M_{8}}\right)$. Van Kampen's theorem implies that

$$
\pi_{1}\left(M_{8}\left(\frac{p}{q}\right)\right)=\Gamma_{8} /\langle\langle\sigma\rangle\rangle
$$

where $\langle\langle\sigma\rangle\rangle$ is the normal closure of $\{\sigma\}$ in $\Gamma_{8}$. 
Now suppose $4 \mid p, 3 \nmid p$. For any positive integer $k$, construct integers $n_{k}, D_{k}$ as follows:

$$
\begin{aligned}
& n_{k}=n_{k}(p, q)=-3\left(p^{2}+12 q^{2}\right)(2+3 k)+9, \\
& D_{k}=D_{k}(p, q)=\left(p^{2}+12 q^{2}\right)\left(n_{k}\right)^{2}+2+3 k .
\end{aligned}
$$

Define a sequence $\left\{\Gamma_{D_{k}}\right\}_{k=1}^{\infty}$ of pairwise noncommensurable, cocompact Fuchsian subgroups $\Gamma_{D_{k}}=\operatorname{Stab}_{\Gamma_{8}}\left(\mathcal{C}_{D_{k}}\right)$ of $\Gamma_{8}$. From Theorem 4.1 we obtain the sequence $\left\{S_{D_{k}}=P_{D_{k}} / \Gamma_{D_{k}} \rightarrow M_{8}\right\}_{k=1}^{\infty}$ of pairwise noncommensurable, closed, orientable, immersed, totally geodesic surfaces in $M_{8}$ with $\pi_{1}\left(S_{D_{k}}\right) \cong \Gamma_{D_{k}}$.

We can now restate a more precise version of Theorem 1.2, the main theorem of [7].

Theorem 4.2 For any integer $p$ such that $4 \mid p, 3 \nmid p$ and $q$ relatively prime to $p$, let $\left\{D_{k}\right\}_{k=1}^{\infty}=\left\{D_{k}(p, q)\right\}_{k=1}^{\infty}$ be as above. Then for every $k$, the closed, immersed, totally geodesic surface $S_{D_{k}} \rightarrow M_{8}$ compresses in $M_{8}(p / q)$.

We note that $D_{k}(p, q)$ depends only on $k$ and $p^{2}+12 q^{2}$. Our approach to prove the main theorem is to show that for a given integer $n$, we can find at least $n$ ways to represent the form $p^{2}+12 q^{2}$, where $p, q$ satisfy the above hypothesis. More precisely, there exists a family $\left\{\left(p_{i}, q_{i}\right)\right\}_{i=1}^{m}$, where $m \geq n$, such that $D_{k}\left(p_{i}, q_{i}\right)=D_{k}\left(p_{j}, q_{j}\right)$ for all $i, j=1, \ldots, m$ and all positive integer $k$. By Theorem 4.2, there are infinitely many closed, immersed, totally geodesic surfaces $S_{D_{k}} \rightarrow M_{8}$ which compress in $M_{8}\left(p_{i} / q_{i}\right)$ for all $i=1, \ldots, m$ and all positive integer $k$. To find such representations $\left\{\left(p_{i}, q_{i}\right)\right\}_{i=1}^{m}$, we will need some facts about quadratic forms.

\section{Quadratic forms}

In this section we recall the relevant facts from number theory and basic properties of Legendre symbol and quadratic forms that will be important tools for the proof of the main theorem (see Cox [3] and Lang [6] for more details).

For any integer $a$ and positive odd prime $p$, the Legendre symbol $(a / p)$ is defined by

$$
(a / p)= \begin{cases}0 & \text { if } p \mid a, \\ +1 & \text { if } p \nmid a \text { and there exists an integer } x \text { such that } x^{2} \equiv a(\bmod p), \\ -1 & \text { otherwise. }\end{cases}
$$

We list here some well-known properties of the Legendre symbol we will need (see Lang [6, pages 77, 78 and 81] for more details). 
Proposition 5.1 Let $p, q$ be distinct, positive, odd primes, and $a, b$ be integers.

(1) (Completely multiplicative law) $(a b / p)=(a / p)(b / p)$.

(2) (Quadratic reciprocity law)

If $p \equiv 1(\bmod 4)$ or $q \equiv 1(\bmod 4)$, then $(p / q)=(q / p)$.

If $p \equiv q \equiv 3(\bmod 4)$, then $(p / q)=(-1)(q / p)$.

(3) (First supplement to the quadratic reciprocity law)

$(-1 / p)=1$ if and only if $p \equiv 1(\bmod 4)$.

Using this, we prove the following.

Lemma 5.2 For any prime $p$ greater than $3,(3 / p)=1$ if and only if $p \equiv 1$ or 11 $(\bmod 12)$.

Proof Any prime $p$ greater than 3 has $p \equiv 1,5,7$ or $11(\bmod 12)$. Given such $p$ apply Proposition 5.1 part (2), and the fact that for any integer $x, x^{2} \equiv 0$ or $1(\bmod 3)$.

A quadratic form $f(x, y)=a x^{2}+b x y+c y^{2}$ is called primitive if its coefficients, $a, b$ and $c$ are relatively prime. We say an integer $m$ is represented by $f(x, y)$ if the equation $m=f(x, y)$ has an integer solution. If this solution has $x$ and $y$ relatively prime, then we say that $m$ is properly represented by $(x, y)$. We declare two primitive forms $f(x, y)$ and $g(x, y)$ to be properly equivalent, and write $f(x, y) \sim g(x, y)$, if there exist integers $p, q, r$ and $s$ such that $f(x, y)=g(p x+q y, r x+s y)$ and $p s-r q=1$. One can easily check that this defines an equivalence relation.

The discriminant of the form $f(x, y)=a x^{2}+b x y+c y^{2}$ is $\mathcal{D}=b^{2}-4 a c$. Direct computation shows that if $f(x, y)=g(p x+q y, r x+s y)$, then $\mathcal{D}_{f}=(p s-q r)^{2} \mathcal{D}_{g}$, where $\mathcal{D}_{f}$ and $\mathcal{D}_{g}$ are the discriminants of the forms $f$ and $g$, respectively. This implies that properly equivalent forms have the same discriminant. We restrict our discussion only to the case $\mathcal{D}<0$, and then $f$ is positive definite. A primitive positive definite form $f(x, y)=a x^{2}+b x y+c y^{2}$ is said to be a reduced form if $|b| \leq a \leq c$, and if $|b|=a$ or $a=c$ then $b \geq 0$.

Each equivalence class has a good representative quadratic form by Lagrange's Theorem of Reduced Forms (see [3, Theorem 2.8]).

Theorem 5.3 Every primitive positive definite form is properly equivalent to a unique reduced form. 
Note that for a fixed discriminant $\mathcal{D}<0$, there are only finitely many reduced forms. Therefore, the number of classes of primitive, positive definite forms of discriminant $\mathcal{D}$ is finite. To see this, consider a reduced form $f(x, y)=a x^{2}+b x y+c y^{2}$. By definition of a reduced form, we have $b^{2} \leq a^{2}, a \leq c$. This implies

$$
-\mathcal{D}=4 a c-b^{2} \geq 4 a^{2}-a^{2}=3 a^{2}
$$

and

$$
0<a \leq \sqrt{\frac{-\mathcal{D}}{3}}, \quad \text { then }|b| \leq a \leq \sqrt{\frac{-\mathcal{D}}{3}} .
$$

Hence there are only finitely many of choices for the integers $a, b$ and $c$. For example we have the following.

Lemma 5.4 There are exactly two reduced forms of discriminant $\mathcal{D}=-48$; namely, $3 x^{2}+4 y^{2}$ and $x^{2}+12 y^{2}$.

Proof From the discussion above, a reduced form $f(x, y)=a x^{2}+b x y+c y^{2}$ of discriminant $\mathcal{D}=-48$ must satisfies $|b| \leq a \leq \sqrt{48 / 3}=4$, and $0 \leq c \leq 16$. An explicit finite search reveal that $3 x^{2}+4 y^{2}$ and $x^{2}+12 y^{2}$ are the only possibilities.

We will need the following theorem of Gauss (see Cox [3, Theorem 3.25] and Landau [5, page 144]).

Theorem 5.5 Let $m$ be a positive odd number relatively prime to $k>1$. Then the number of ways that $m$ is properly represented by a reduced form of discriminant $-4 k$ is

$$
2 \prod_{p \mid m}\left(1+\left(\frac{-k}{p}\right)\right),
$$

where the product is over all distinct positive prime divisors $p$ of $m$.

This theorem allows us to prove the following.

Theorem 5.6 For a given positive integer $m \equiv 7(\bmod 12)$ with all prime divisors congruent to 1 or $7(\bmod 12)$, the number of proper representations of $m$ by the primitive positive form $3 x^{2}+4 y^{2}$ is $2^{\tau(m)+1}$ where $\tau(m)$ is the number of positive prime divisors of $m$.

Proof Let us first investigate the properties of $m$. Observe that $m \equiv 7(\bmod 12)$ implies that $m$ is odd and $m \equiv 3(\bmod 4)$. Since 3 is not a square $\bmod 4, m$ cannot be properly represented by the form $x^{2}+12 y^{2}$. 
With the given conditions on the divisors of $m$, we can write

$$
m=p_{1}{ }^{\alpha_{1}} \cdots p_{s}^{\alpha_{s}} q_{1} \beta_{1} \cdots q_{t}{ }^{\beta_{t}}
$$

where $p_{1}, \ldots, p_{s}$ are distinct positive primes congruent to $1(\bmod 12)$ and $q_{1}, \ldots, q_{t}$ are distinct positive primes congruent to $7(\bmod 12)$. Then

$$
7 \equiv m \equiv 7^{\beta_{1}+\cdots+\beta_{t}} \quad(\bmod 12)
$$

and it follows that

$$
\beta_{1}+\cdots+\beta_{t} \equiv 1 \quad(\bmod 2) .
$$

Now we consider the proper equivalence classes of the fixed discriminant -48 . By Lemma 5.4, there are exactly 2 classes of primitive, positive definite reduced forms $3 x^{2}+4 y^{2}$ and $x^{2}+12 y^{2}$. However, as noted above, $m$ cannot be represented by the latter form. Therefore, appealing to Theorem 5.5, Proposition 5.1 and Lemma 5.2, the number of ways that $m$ is properly represented by a reduced form $3 x^{2}+4 y^{2}$ is

$$
\begin{aligned}
2 \prod_{p \mid m}\left(1+\left(\frac{-12}{p}\right)\right) & =2 \prod_{p \mid m}\left(1+\left(\frac{-3}{p}\right)\left(\frac{4}{p}\right)\right) \\
& =2 \prod_{i=1}^{s}\left(1+\left(\frac{-3}{p_{i}}\right)\right) \prod_{i=1}^{t}\left(1+\left(\frac{-3}{q_{i}}\right)\right) \\
& =2 \prod_{i=1}^{s}\left(1+\left(\frac{-1}{p_{i}}\right)\left(\frac{3}{p_{i}}\right)\right) \prod_{i=1}^{t}\left(1+\left(\frac{-1}{q_{i}}\right)\left(\frac{3}{q_{i}}\right)\right) \\
& =2 \prod_{i=1}^{s}(1+(1)(1)) \prod_{i=1}^{t}(1+(-1)(-1)) \\
& =2^{(s+t)+1}=2^{\tau(m)+1} .
\end{aligned}
$$

As a consequence, we have the following corollary.

Corollary 5.7 Let $N=4 m$ for some positive integer $m$ such that $m \equiv 7(\bmod 12)$ and all prime divisors of $m$ are congruent to 1 or $7(\bmod 12)$. The number of ways to properly represent $N$ in the form $N=p^{2}+12 q^{2}$, where $4 \mid p$ and $3 \nmid p$, is exactly $2^{\tau(N)}$.

Proof Writing $p=4 r$ sets up to bijection between the proper representations of $N=p^{2}+12 q^{2}$ and $m=3 q^{2}+4 r^{2}$. So it suffices to prove that the number of ways to properly represent $m=3 q^{2}+4 r^{2}$ is $2^{\tau(N)}=2^{\tau(m)+1}$.

Applying Theorem 5.6, the number of ways to represent $m=3 q^{2}+4 r^{2}$ is exactly $2^{\tau(m)+1}$, as required. 


\section{Compression}

In this section we prove:

Theorem 6.1 Given any positive integer $n$, there exist $n$ distinct slopes $\alpha_{1}, \ldots, \alpha_{n}$ in the $\partial M_{8}$ and infinitely many closed, orientable, immersed, incompressible surfaces $S_{D_{k}} \uparrow \rightarrow M_{8}$ with no incompressible annulus joining $S_{D_{k}}$ and $\partial M_{8}$ which compress in $M_{8}\left(\alpha_{i}\right)$ for all $i=1, \ldots, n$ and positive integer $k$.

From this, Theorem 1.1 easily holds.

Proof of Theorem 1.1 By assuming Theorem 6.1, this theorem immediately follows when we let $M=\bar{M}_{8}$ which is a compact, orientable, irreducible 3-manifold with torus boundary. We note that $M(\alpha)=M_{8}(\alpha)$ for any slope $\alpha$ in $\partial M$.

To prove Theorem 6.1 we first recall Dirichlet's Theorem on arithmetic progressions (see Selberg [15] for more details).

Theorem 6.2 If positive numbers $s$ and $t$ are relatively prime, then there are infinitely many primes $p$ such that $p \equiv s(\bmod t)$.

Using this, we can prove:

Lemma 6.3 For any given positive integer $n$, there is a family of $n$ pairs $\left\{\left(p_{i}, q_{i}\right)\right\}_{i=1}^{n}$ such that $p_{i}^{2}+12 q_{i}^{2}=p_{j}^{2}+12 q_{j}^{2}$ for all $i, j=1, \ldots, n$, where $p_{i}$ and $q_{i}$ are relatively prime, $4 \mid p_{i}$, but $3 \nmid p_{i}$ for all $i=1, \ldots, n$.

Proof For any given positive integer $n$, there exists a positive integer $k$ such that $n \leq 2^{k}$. Define the integer $N$ by

$$
N=4 p_{1}^{\alpha_{1}} \cdots p_{s}^{\alpha_{s}} q_{1}^{\beta_{1}} \cdots q_{t}^{\beta_{t}}
$$

where $s+t=k, p_{1}, \ldots, p_{s}$ are distinct positive primes congruent to $1(\bmod 12)$, $q_{1}, \ldots, q_{t}$ are distinct positive primes congruent to $7(\bmod 12)$ and $\beta_{1}+\cdots+\beta_{t} \equiv 1$ $(\bmod 2)$. Since 1 and 7 are both relatively prime to 12 , we know that such integer $N$ exists by Dirichlet's theorem.

By construction, $N$ satisfies the hypothesis of Corollary 5.7. Therefore, there exist $2^{\tau(N)}=2^{k} \geq n$ pairs $(p, q)$ which properly represent $N$, and moreover these satisfy the conditions of the lemma. 
Example 6.4 Using Mathematica for $n=16$, we have the family

$\{(32,813),(200,811),(680,789),(1112,747),(1328,717),(1528,683)$,

$(1640,661),(1912,597),(2032,563),(2320,461),(2560,339),(2608,307)$,

$(2648,277),(2720,211),(2752,173),(2792,107)\}$.

All pairs $(p, q)$ are relatively prime, $4 \mid p, 3 \nmid p$ and $p^{2}+12 q^{2}=7,932,652=$ $2^{2} \cdot 7 \cdot 13 \cdot 19 \cdot 31 \cdot 37$.

Note that there are 16 pairs $(p, q)$ here, but $2^{\tau(N)}=64$. This because each pair $(p, q)$ determines 4 distinct pairs; $(p, q),(-p, q),(p,-q),(-p,-q)$.

Proof of Theorem 6.1 For any given $n$, by Lemma 6.3 there is a family $\left\{\left(p_{i}, q_{i}\right)\right\}_{i=1}^{2 n}$ such that $p_{i}^{2}+12 q_{i}^{2}=p_{j}^{2}+12 q_{j}^{2}$ for all $i, j=1, \ldots, 2 n$, where $p_{i}$ and $q_{i}$ are relatively prime such that $4 \mid p_{i}$, but $3 \nmid p_{i}$ for all $i=1, \ldots, 2 n$. For each $i=1, \ldots, 2 n$, consider the slope $p_{i} / q_{i}$ on $\partial M_{8}$, because $p_{i} / q_{i}=-p_{i} /-q_{i}$, this determines at least $n$ distinct slopes call them $\alpha_{1}, \ldots, \alpha_{n}$. As noted in Section 4.2, $D_{k}\left(p_{i}, q_{i}\right)=D_{k}\left(p_{j}, q_{j}\right)$ for all $i, j=1, \ldots, 2 n$ and all $k>0$, and we denote this simply as $D_{k}$. Theorem 4.2 implies $S_{D_{k}}$ compresses in $M_{8}\left(\alpha_{i}\right)$ for all $i=1, \ldots, n$ and all $k>0$. Since $D_{1}<D_{2}<\cdots$, Theorem 4.1 implies $S_{D_{k}}$ are all noncommensurable.

\section{References}

[1] A Bart, Surface groups in some surgered manifolds, Topology 40 (2001) 197-211 MR1791272

[2] R Benedetti, C Petronio, Lectures on hyperbolic geometry, Universitext, Springer, Berlin (1992) MR1219310

[3] D A Cox, Primes of the form $x^{2}+n y^{2}$. Fermat, class field theory and complex multiplication, Wiley-Interscience Publ., Wiley, New York (1989) MR1028322

[4] M Culler, C M Gordon, J Luecke, P B Shalen, Dehn surgery on knots, Ann. of Math. (2) 125 (1987) 237-300 MR881270

[5] E Landau, Vorlesungen über Zahlentheorie. I, Hirzel, Leipzig (1927)

[6] S Lang, Algebraic number theory, second edition, Graduate Texts in Math. 110, Springer, New York (1994) MR1282723

[7] C J Leininger, Compressing totally geodesic surfaces, Topology Appl. 118 (2002) 309-328 MR1874553

[8] D Long, A W Reid, Surface subgroups and subgroup separability in 3-manifold topology, 25 o Colóquio Brasileiro de Matemática, IMPA, Rio de Janeiro (2005) MR2164951 
[9] C Maclachlan, Fuchsian subgroups of the groups $\operatorname{PSL}_{2}\left(O_{d}\right)$, from: "Low-dimensional topology and Kleinian groups (Coventry/Durham, 1984)”, (D B A Epstein, editor), London Math. Soc. Lecture Note Ser. 112, Cambridge Univ. Press (1986) 305-311 MR903873

[10] C Maclachlan, A W Reid, The arithmetic of hyperbolic 3-manifolds, Graduate Texts in Math. 219, Springer, New York (2003) MR1937957

[11] J G Ratcliffe, Foundations of hyperbolic manifolds, second edition, Graduate Texts in Math. 149, Springer, New York (2006) MR2249478

[12] A W Reid, Arithmetic Kleinian groups and their Fuchsian subgroups, PhD thesis, University of Aberdeen (1987)

[13] R Riley, A quadratic parabolic group, Math. Proc. Cambridge Philos. Soc. 77 (1975) 281-288 MR0412416

[14] D Rolfsen, Knots and links, Math. Lecture Series 7, Publish or Perish, Houston, TX (1990) MR1277811 Corrected reprint of the 1976 original

[15] A Selberg, An elementary proof of Dirichlet's theorem about primes in an arithmetic progression, Ann. of Math. (2) 50 (1949) 297-304 MR0029409

[16] W P Thurston, The geometry and topology of three-manifolds, Princeton Univ. Math. Dept. Lecture Notes (1979) Available at http://msri.org/publications/books/ gt $3 \mathrm{~m} /$

[17] Y Q Wu, Incompressibility of surfaces in surgered 3-manifolds, Topology 31 (1992) 271-279 MR1167169

[18] Y-Q Wu, Immersed essential surfaces and Dehn surgery, Topology 43 (2004) 319-342 MR2052966

[19] Y-Q Wu, Depth of pleated surfaces in toroidal cusps of hyperbolic 3-manifolds, Algebr. Geom. Topol. 9 (2009) 2175-2189 MR2551666

Department of Mathematics, University of Illinois at Urbana-Champaign 1409 W Green Street, Urbana IL 61801, USA

Department of Mathematics, Science Faculty, Chiangmai University

Chiangmai 50200, Thailand

jaipong2@illinois.edu

Received: 3 September $2010 \quad$ Revised: 7 November 2010 